\title{
Multidrug efflux pumps: drug binding - gates or cavity?
}

\author{
Emily Crowley and Richard Callaghan \\ Nuffield Department of Clinical Laboratory Sciences, University of Oxford, UK
}

\author{
Keywords \\ central cavity; coupling; domain interface; \\ drug binding; drug resistance; drug \\ transport; interdomain communication; \\ membrane protein; multidrug binding; \\ P-glycoprotein

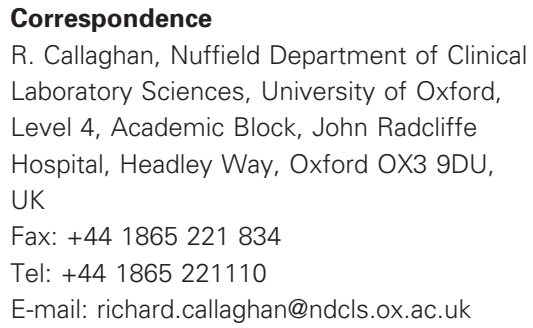

(Received 22 July 2009, revised 1 October 2009, accepted 5 November 2009)
The role of the ATP-binding cassette ABCB1 in mediating the resistance to chemotherapy in many forms of cancer has been well established. The protein is also endogenously expressed in numerous barrier and excretory tissues, thereby regulating or impacting on drug pharmacokinetic profiles. Given these prominent roles in health and disease, a great deal of biochemical, structural and pharmacological research has been directed towards modulating its activity. Despite the effort, only a small handful of compounds have reached the later stages of clinical trials. What is responsible for this poor return on the heavy research investment? Perhaps the most significant factor is the lack of information on the location, physical features and chemical properties of the drug-binding site(s) in ABCB1. This minireview outlines the various strategies and outcomes of research efforts to pin-point the sites of interaction. The data may be assimilated into two working hypotheses to describe drug binding to ABCB1; (a) the central cavity and the (b) domain interface models.

\section{Properties of drug-binding sites in ABCB1}

P-Glycoprotein (also known as ABCB1, mdr1) has long been associated with drug resistance in many cancer types. This protein is a member of the ATPbinding cassette (ABC) family and is believed to confer drug resistance in cancer cells by mediating the active outward efflux of chemotherapeutic drugs. Providing information on the nature of the drug-ABCB1 interaction has been a vital and synergistic pursuit alongside efforts to locate the drug-binding sites on the protein. The range and number of compounds 'recognized' by $\mathrm{ABCB} 1$ are astounding and there remains no conclusive explanation for this polyspecific behaviour. Numerous pharmacological studies utilizing equilibrium drug binding, hydrolysis of ATP and steady-state accumulation assays have demonstrated that ABCB1 contains multiple sites for drug interaction. Moreover, complex allosteric interactions between sets of drugs have been demonstrated which may involve negative heterotrophy or conversely, permit the simultaneous binding of two drugs [1-4]. ABCB1 displays a complex mechanism of drug translocation across the membrane that requires coupling between the energy-providing nucleotide-binding domains (NBDs) and the transmembrane domains (TMDs), which contain the drugbinding sites and the translocation conduit. There is considerable debate concerning the characteristics of coupling, specifically whether one or two ATP molecules are hydrolysed per transport event and whether ATP binding per se or the hydrolytic step is responsible for switching the binding site orientation during

\section{Abbreviations}

ABC, ATP-binding cassette; ABCB1, the multidrug resistance P-glycoprotein; IAAP, iodoarylazidoprazosin; NBD, nucleotide-binding domain; $\mathrm{TM}$, transmembrane; TMD, transmembrane domain. 
transport. Binding events are also not equal because some interaction of certain compounds leads to transport and the stimulation of hydrolysis, whereas others are not transported and cause potent inhibition of ATPase activity. Elucidating the location and biophysical properties of the drug-binding sites would provide considerable input into the molecular interaction between substrates/inhibitors with ABCB1. Structural and pharmacological information of this ilk would facilitate the development of potent inhibitors of $\mathrm{ABCB} 1$ to restore the effectiveness of many anticancer agents.

\section{Strategies to locate the drug-binding sites}

The inability to precisely localize the drug-binding sites on $\mathrm{ABCB} 1$ has not been caused by a lack of effort. Since 1989, a large number of research teams have employed numerous strategies to elucidate the precise location of drug-binding sites on $\mathrm{ABCB} 1$ and a summary of the major findings is presented in Table 1. This not an exhaustive list, but does highlight many significant observations during the past two decades.

Initial attempts made use of photo-active versions of $\mathrm{ABCB} 1$ inhibitors including $\left.{ }^{125} \mathrm{I}\right]$ - iodoarylazidoprazo$\sin$ (IAAP) and $\left[{ }^{3} \mathrm{H}\right]$-azidopine [5-7]. Typically, the drug was covalently attached to the protein, which was then subjected to either protease digestion or chemical cleavage. Protein fragments containing bound drug were analysed using specifically generated antibodies to identify the binding site. A more recent investigation employed a similar strategy using a propafenone derivative to probe drug interaction [8]. The protein was chemically cleaved and the fragments analysed by MALDI-TOF MS. This enabled precise identification of the fragments labelled by drug and enabled relative quantitation of the amount of drug bound.

Resistant cell lines expressing $\mathrm{ABCB} 1$ may be generated by the long-term exposure of cells to anticancer drugs. In the presence of high drug concentrations, this strategy frequently produced mutations within the protein, many concentrated in the TMDs [9]. Mutated forms of $\mathrm{ABCB} 1$ conferred a distinct resistance profile to the wild-type protein, which was thought to reflect alterations in drug binding or transport. Subsequent, more sophisticated, studies used directed mutagenesis to introduce mutations into targeted regions of the protein [10-12]. The functional consequences of the mutations were assessed using a range of assays including the ability of $\mathrm{ABCB} 1$ to confer cellular resistance, reduce intracellular drug accumulation, bind drug and/or display drug-stimulated ATPase activity. Frequently, a range of these assays was employed to provide a more detailed understanding of the contribution of specific residues to protein activity. A popular approach involved the mutagenesis of target residues to cysteine, which enables conjugation of thiol-reactive drug derivatives and exploration of the local environment and topography [13-16].

The current structural resolution of $\mathrm{ABCB} 1$ does not provide atomic details on the drug-binding site. However, structural information is available for a number of bacterial ABC transporters including one (Sav1866) that is likely to act as a multidrug efflux pump [17-19]. Consequently, a number of in silico molecular models of $\mathrm{ABCB} 1$ have been developed based on these structures. Several teams have attempted to 'dock' drugs to the structure in the hope of identifying likely regions involved in the drug-protein interaction.

\section{What regions are implicated in binding?}

This minreview is not intended to provide an extensive description of the past two decades' research into the drug-binding sites of ABCB1. Overall, the studies highlighted in Table 1 can be divided into two broad descriptions of the drug-binding sites: the central cavity model and the domain interface model.

\section{The central cavity model}

The ongoing electron microcopy-structure investigations were the first to reveal that $\mathrm{ABCB} 1$ has a central cavity which is likely to be aqueous filled and that the TMDs have regions of discontinuity that may allow access to the lipid milieu [20-24]. A number of biochemical studies suggest that the transmembrane (TM) helices lining this central cavity contribute to drug binding [25].

The absence of a high-resolution structure (i.e. at or better than $3 \AA$ ) hampers the assignment of specific helices and their constitutive residues involved in lining the central pore. A series of investigations has attempted to produce a topographical map of the central cavity by covalent cross-linking of cysteines introduced into prospective helices [13,26,27]. Cross-linking agents of different lengths have been used to generate a dimensional aspect to the map. Interestingly, formation of several of the cross-links is perturbed by the presence of substrates or inhibitors of $\mathrm{ABCB} 1$; this has been suggested to demonstrate that the residues implicated in cross-link formation mediate drug interactions. Moreover, the ability to form long-distance cross-links has also been interpreted as evidence that the residues are located on the helical face in contact with the central cavity. 
Table 1. Time-line of the search for the location of drug-binding sites on P-glycoprotein. The time-line contains a summary of the strategies and observations obtained from studies specifically aimed at locating the sites of drug binding to P-glycoprotein. The time-line shows a selection of the major advances in this area and does not include reference to numerous studies describing the nature or physicochemical properties of the binding sites. DBS, drug binding site; EC, extracellular; IAAP, iodoarylazidoprazosin; NBD, nucleotide-binding domain; TMD, transmembrane domain.

\begin{tabular}{|c|c|c|}
\hline Date & Reference & Comments \\
\hline \multirow[t]{2}{*}{1989} & [5] & Strategy: Photoaffinity labelling and protein digestion \\
\hline & & Result: Two sites or one with components in each half \\
\hline \multirow[t]{2}{*}{ 1990-1991 } & {$[7,52]$} & Strategy: Labelling, digestion and antibody-mediated identification \\
\hline & & Result: 6 kDa fragment labelled within/close to TM11 and TM12 \\
\hline \multirow[t]{2}{*}{1993} & [10] & Strategy: Phe $\rightarrow$ Ser mutations within TM11 followed by transport/cytotoxicity assays \\
\hline & & Result: TM11 contributes to DBS \\
\hline \multirow[t]{2}{*}{1993} & {$[6]$} & Strategy: Labelling, digestion and antibody-mediated identification \\
\hline & & Result: Labelling at two regions C-terminal to TM6/12 \\
\hline \multirow[t]{2}{*}{1993} & [11] & Strategy: Phe $\rightarrow$ Ala mutations at nucleotides 335 (TM6) and 978 (TM12) \\
\hline & & $\begin{array}{l}\text { Result: Mutations altered resistance profile, suggesting that TM6/12 contributes to binding and/or } \\
\text { translocation }\end{array}$ \\
\hline \multirow[t]{2}{*}{1994} & [53] & Strategy: Theoretical - molecular simulations \\
\hline & & $\begin{array}{l}\text { Result: Proposed that drugs intercalate between multiple Phe residues. Several helices contain Phe } \\
\text { residues which shields drug from the aqueous environment - implicate TM3, TM5, TM8 and TM11 } \\
\text { forming DBS }\end{array}$ \\
\hline \multirow[t]{2}{*}{1995} & [54] & Strategy: $\mathrm{ABCB} 1$ chimera assessed by cytotoxicity and photolabelling \\
\hline & & $\begin{array}{l}\text { Result: Loop between TM11 and TM12 (EC0) modulates resistance spectrum \& may be involved in } \\
\text { translocation pathway }\end{array}$ \\
\hline \multirow[t]{2}{*}{1997} & [12] & Strategy: Site-directed mutagenesis and cytotoxicity \\
\hline & & $\begin{array}{l}\text { Result: Mutations in TM6 alter the ability of cyclosporin A (not verapamil) to overcome resistance; TM6 } \\
\text { involved in selectivity }\end{array}$ \\
\hline \multirow[t]{2}{*}{1997} & [55] & Strategy: Photolabelling and protein digestion \\
\hline & & $\begin{array}{l}\text { Result: Differential effects of flupentixol on two [ }{ }^{125} \text { I]-IAAP labelling sites - nonidentical binding sites in } \\
\text { N- \& C-termini }\end{array}$ \\
\hline \multirow[t]{2}{*}{1998} & [56] & Strategy: Chemical structure-activity relationships for substrates \\
\hline & & Result: $\mathrm{H}$-bonding patterns in substrates are key elements in drug recognition \\
\hline \multirow[t]{2}{*}{1998} & [57] & Strategy: Site-directed mutagenesis of TM12 \\
\hline & & Result: N-terminal region of TM12 influences transport specificity \\
\hline \multirow[t]{2}{*}{2001} & [58] & Strategy: IAAP labelling and chemical cleavage \\
\hline & & $\begin{array}{l}\text { Result: Three regions of labelling found; TM4-5, TM7-8 and post-NBD2. Single site for IAAP comprising } \\
\text { multiple spatial elements }\end{array}$ \\
\hline \multirow[t]{2}{*}{2001} & [59] & Strategy: Effects of TM9 mutations on cytotoxicity and photolabelling \\
\hline & & $\begin{array}{l}\text { Result: Mutations in TM9 produce a distinct resistance pattern similar to TM6. TM9 and TM6 may } \\
\text { co-operate in IAAP labelling }\end{array}$ \\
\hline \multirow[t]{3}{*}{2005} & [8] & Ligand: $\left[{ }^{3} \mathrm{H}\right]$ propafenone and analogues \\
\hline & & Strategy: Photolabelling and identification with MALDI-TOF MS \\
\hline & & $\begin{array}{l}\text { Result: Two binding regions - TM3/11 and TM5/8. MsbA-based model suggest the two sites are at } \\
\text { TMD : TMD interfacial regions }\end{array}$ \\
\hline \multirow[t]{2}{*}{2005} & {$[2,60]$} & Strategy: Mapping R/H sites; fluorescence approach \\
\hline & & $\begin{array}{l}\text { Result: H-site within bilayer leaflet region of ABCB1, whereas the R-site is in the cytosolic region. The } \\
\text { R-site can bind two drugs simultaneously }\end{array}$ \\
\hline \multirow[t]{2}{*}{2005} & [61] & Strategy: Review of their site-directed mutagenesis studies \\
\hline & & $\begin{array}{l}\text { Result: Suggest a common drug-binding site in the central cavity Interfaces at TM5/8 and TM2/11 form } \\
\text { gates to the cavity and drugs negotiate passage through these gates }\end{array}$ \\
\hline \multirow[t]{2}{*}{2006} & [36] & Strategy: Theoretical study - MsbA-based ABCB1 model \\
\hline & & $\begin{array}{l}\text { Result: Propose a large central binding cavity with a lateral opening to lipid bilayer. Cavity helices include } \\
\text { TM4, TM5, TM6, TM10, TM11 and TM12 }\end{array}$ \\
\hline \multirow[t]{2}{*}{2006} & {$[62,63]$} & Strategy: Directed mutagenesis and drug labelling \\
\hline & & Result: Two studies suggesting that TM $1 / 7$ also contribute to the binding pocket in the central cavity \\
\hline \multirow[t]{2}{*}{2006} & [64] & Strategy: Simulations to probe drug-ABCB1 interaction \\
\hline & & $\begin{array}{l}\text { Result: Argue that those residues at the interfacial region and that this is in contact with the polar head } \\
\text { group region of the membrane }\end{array}$ \\
\hline \multirow[t]{2}{*}{2007} & [35] & Strategy: Sav186-based model used to characterize drug binding \\
\hline & & Result: Proposed several key residues from TM1, TM5 and TM6 in drug binding \\
\hline
\end{tabular}


Table 1. (Continued)

\begin{tabular}{lll}
\hline Date & Reference & Comments \\
\hline 2007 & {$[15,16,30]$} & $\begin{array}{l}\text { Strategy: Cysteine-directed mutagenesis of TM6 } \\
\text { Result: Mutations did not alter initial drug binding however the helix was crucial in mediating TMD-NBD } \\
\text { communication }\end{array}$ \\
& & $\begin{array}{l}\text { Strategy: MsbA-based model to dock drugs onto } \\
\text { Result: Proposed a number of binding clusters dotted throughout the TMDs and that multiple drugs could } \\
\text { interact simultaneously }\end{array}$ \\
\hline
\end{tabular}

Mutations of several residues suggested to line the central cavity have been independently shown to alter $\mathrm{ABCB} 1$ function. Perhaps the most frequent reported manifestation is an alteration in the cytotoxicity profile - for example, increased resistance conferred to actinomycin D [9]. A change in the resistance profile may indicate that the residue in question favours interaction with one drug over another. Other reported manifestations are altered steady-state accumulation of ABCB1 substrates within cells and modified stimulation of the basal rate of ATP hydrolysis. These alterations in $\mathrm{ABCB} 1$ function have also been interpreted in terms of the initial drug-protein binding event.

The strategy of introducing cysteine residues throughout the cavity-lining helices has yielded considerable, but often contradictory, mechanistic information on helical involvement in ABCB1 function. One approach has been to ascertain the propensity of the introduced cysteine to react with a maleimide-drug (i.e. thiol reactive) conjugate $[14,28]$. Covalent attachment of the drug conjugate was reasoned to demonstrate that the residue was located within the drug-binding pocket. The data were supported by the prevention of cysteine attachment using a drug without the maleimide moiety. Other investigations have focused on the consequences of the cysteine mutation per se, or following attachment of thiol-reactive, nonsubstrate, probe molecules $[15,29,30]$. This approach also allowed investigation of the helical topography and how this alters during functionally relevant conformational changes in the protein.

The studies discussed above have been ongoing for several years and involve many independent research teams. It appears that a large number of helices (TM1, TM4-7 and TM9-12) may line the central cavity, contribute to the drug-binding pocket and/or regulate conformational coupling within $\mathrm{ABCB} 1$. It is worth noting that TM6 and TM12 have consistently been implicated in important functional roles for ABCB1. The number of TM helices ( 9 of 12) predicted to line the central cavity suggests a large dimension. Not all residues within the cavity-lining helices demonstrate a strong functional role and furthermore, certain mutations show selectivity towards different substrate molecules. A central, often aqueous filled, cavity is not limited to ABCB1 because structural studies with a number of $\mathrm{ABC}$ transporters have revealed similar findings $[17,31,32]$.

Collectively, these data may be consistent with a large binding domain or pocket and the presence of a drug imparting distinct conformational alterations akin to the 'induced fit' model for drug binding.

\section{The domain interface model}

As is the case for all $\mathrm{ABC}$ transport proteins, the membrane-spanning region of $\mathrm{ABCB} 1$ comprises two domains. Both halves of the TMD appear to be functionally important and capable of drug interaction, a point that is strongly supported by studies that photolabel ABCB1, digest the protein and identify fragments containing the attached drug (Table 1). The electron microscopy structures for $\mathrm{ABCB} 1$ display a discontinuity in the TMD region; however, resolution of the data does not yet enable prediction of the proximal helices. A number of cross-linking studies have focused on generating a spatial topology map for $\mathrm{ABCB} 1$ and a similar aim has been targeted through molecular models based on the high-resolution structures of Sav186 and MsbA [33-36]. The consensus appears to be that one of the domain interfaces is mediated by TM5/8, whereas the other comprises TM11/3 with TM2 potentially contributing.

Cysteines introduced at these two interfacial regions demonstrated avid accessibility to conjugation with drug-maleimide compounds, suggesting an involvement in drug binding. However, addition of drug without maleimide did not offer protection against chemical modification. Perhaps the most significant support for the interfacial region comprising a drugbinding site was obtained using the photoactive propafenone derivative $\left[{ }^{3} \mathrm{H}\right]$ GPV51 [8]. Following labelling and chemical digestion, the fragments were analysed for the presence of drug using MALDI-TOF MS. This powerful approach also provided data on the labelling density and indicated that fragments from TM3, TM5, 
TM8 and TM11 accounted for $>70 \%$ of the total bound drug. Based on homology modelling and crosslinking data, it was reasoned that this labelling occurred in two pockets comprising TM5/8 and $\mathrm{TM} 3 / 11$, and that these provided the interface between the $\mathrm{N}$ - and $\mathrm{C}$-terminal halves of the TMDs.

\section{How convincing are the data used to identify the binding sites?}

Considerable debate on the merits and applicability of the two models continues to rage and remains unresolved. Is the experimental data stronger for either model and are the models mutually exclusive? Indeed, there are a number of issues with the supporting data for both models. More correctly, it is interpretation of the supporting data that requires further consideration or refinement. This section highlights many of the drawbacks or limitations of the data currently in use to locate the drug-binding sites on ABCB1.

The resistance profile of cells expressing $\mathrm{ABCB} 1$ is a frequently used reporter of activity and has been employed to infer the functional consequences of mutations in the protein $[10,12]$. However, cytotoxicity assays inform on a whole phenotype and this often means multiple mechanisms of resistance, particularly in the case of cell lines selected in the presence of high anticancer drug concentrations. The precise quantitative contribution of $\mathrm{ABCB} 1$ to the phenotype is difficult to assess in such a complex system. Other functional assays used in whole cells or proteoliposomes include steady-state drug accumulation or stimulation of ATP hydrolysis [37-39]. Drug transport is a multistep process involving drug binding, ATP hydrolysis and conformational changes leading to transbilayer movement. Therefore, attributing altered levels of transport specifically to the initial drug-binding step is difficult to do with conviction. ATPase activity and its stimulation or inhibition by drugs are also complex, involving considerably more than simply drug binding. Perhaps the most conclusive data are provided by directly measuring the drug-ABCB1 interaction using equilibrium binding assays or photoaffinity labelling procedures $[1,3,40]$. Moreover, the greatest confidence may only be afforded by extensive dose-response analyses to measure capacity and affinity changes in drug binding, rather than reliance on a single drug concentration.

Often the pharmacology assays examining ABCB1 function rely on modified versions of the drug; for example, photoactive azide derivatives or drug-maleimide conjugates. Drug derivatives require that the active moiety (e.g. azide) does not interfere with the 'normal' drug-protein interaction. In other words, they must lie outside the actual drug pharmacophore otherwise the true binding affinity or process is not being examined. Another drawback of these derivatives is that the active moiety may exhibit considerable motion. This issue was validated for the interaction of azidopine with L-type calcium channels, wherein the azide moiety exhibited a conical range of motion from the point of contact with the protein [41]. Consequently, the region labelled on the protein may be distinct from the actual binding site or be located at multiple sites.

Many strategies to locate the binding sites involve digestion of $\mathrm{ABCB} 1$ following labelling with reactive drugs. Unfortunately, neither chemical nor proteolytic digestion is complete and therefore frequently displays a heterogeneous fragmentation pattern. Reproducibility issues have also been noted and the combination renders the identification of fragments a difficult task. Early attempts favoured the use of antibodies to identify drug-containing fragments, but generating antibodies for all the fragments produced is an unlikely scenario, particularly for shorter fragments [5-7]. The recent work of Pleban et al. [8] developed a MALDITOF MS approach to circumvent these issues and it certainly warrants greater usage.

ABCB1 adopts numerous stable conformations with the impetus for most of the transitions caused by nucleotide binding/hydrolysis and drug binding. Consequently, labelling or binding conditions need to be carefully controlled to prevent the final data representing an 'averaged' and potentially noninitial conformation. For example, cross-linking between helices in the presence or absence of drug substrates is sensitive to protein movement within the TMDs and may reflect allosteric changes rather than simple steric inhibition of cross-linkage. Similarly, protection assays using unlabelled compound (see previous section) may be affected. The labelled and unlabelled drugs could conceivably bind at distinct sites and the latter may simply cause a conformational change in $\mathrm{ABCB} 1$ that alters the accessibility of the target cysteine residue.

Molecular modelling approaches in the analysis of $\mathrm{ABCB} 1$ are currently based on non-ABCB1 structures and therefore a degree of caution is prudent [33-36]. Two principle structures are employed, namely MsbA and Sav186, but unfortunately these proteins display considerable differences. MsbA, particularly in the basal configuration, has large separation between the two monomers and there is considerable debate on the physiological significance of the various conformations. In comparison, Sav186 displays considerable domain swapping between the two monomers, although this 
finding has not yet been validated biochemically. Are either or both structures correct or do they represent crystal artefacts? More importantly in respect to this minireview, how similar is the structure of $\mathrm{ABCB} 1$ ? Until such discrepancies are reconciled, interpretation of these models can only be considered speculative. Several attempts at docking drugs onto the models have been attempted $[33,35,36]$. However, without absolute knowledge of the drug-binding site location or the features of the drug-ABCB1 interaction being fully described, the data from modelling approaches also need careful and cautious interpretation.

Recently, the structure of mouse $\mathrm{ABCB} 1$ has been obtained using X-ray crystallography and although this is a significant breakthrough, structural resolution was at $3.8 \AA$ in the absence of drug. Structural information was also obtained by 'soaking' crystals in the presence of an $\mathrm{ABCB} 1$ inhibitor and the resolution obtained was slightly lower at $4.4 \AA$. The cyclic hexapeptide inhibitor was synthesized specifically for this study and its relationship to the established pharmacological drug interactions sites (e.g. site I for vinblastine) is presently unclear. In addition, the current level of resolution precludes atomic detail on the drugprotein interaction. Many of the residues implicated in binding to the custom-built inhibitor are, however, equivalent to those from the biochemical investigations. A great deal more analysis and functional investigation based on this structure are required and presumably underway. As the resolution undoubtedly improves towards, or better than, $3 \AA$ so too will our understanding of the molecular interaction of drugs with ABCB1. In particular, we need information on the forces mediating drug binding (e.g. hydrogen bonding), the local solute environment (e.g. pH) and the dimensions or topography of the binding site.

\section{Can the data be reconciled into a map of the sites?}

Where do we stand on the issue of the location of drug-binding sites on ABCB1? There is clearly a wealth of information implicating several regions of the protein (Fig. 1). However, the previous section appears to cast doubt on the findings. This is not meant to be the case, but simply to urge some degree of caution and care in data interpretation. Figure 1 also highlights the fact that a considerable proportion of the protein is involved in drug binding and/or mediating communication pathways between the TMDs and the NBDs. This section aims to reconcile the data into a working model of drug interaction with $\mathrm{ABCB} 1$.

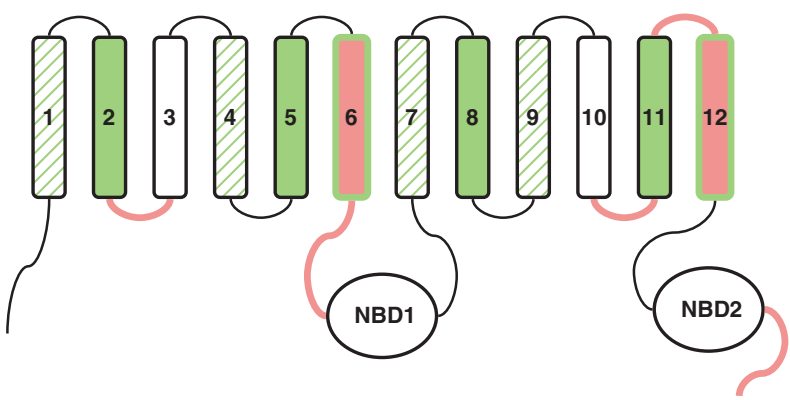

Fig. 1. Topological map of the regions of $A B C B 1$ implicated in drug binding. Schematic depiction of the topological organization of ABCB1 with the rectangular TM helices and circular NBDs. Areas shaded in light green indicate regions of the protein thought to mediate drug binding. The deeper shade of green indicates a greater amount of observational data supporting this role. Pink shading reveals areas of the protein thought to mediate communication pathways involved in the translocation process.

Overall, the data and observations are weighted towards (but not exclusively) three pairs of helices (TM3/11, TM5/8 and TM6/12) playing a significant role in drug binding. The TM6/12 pair is clearly involved in the translocation process, but its precise role is not yet fully resolved. Mutations in these helices alter cytotoxicity profiles, overall drug accumulation and ATP hydrolysis. The controversy relates to the involvement in drug binding per se. Studies with drugmaleimide conjugates favour a role, whereas recent

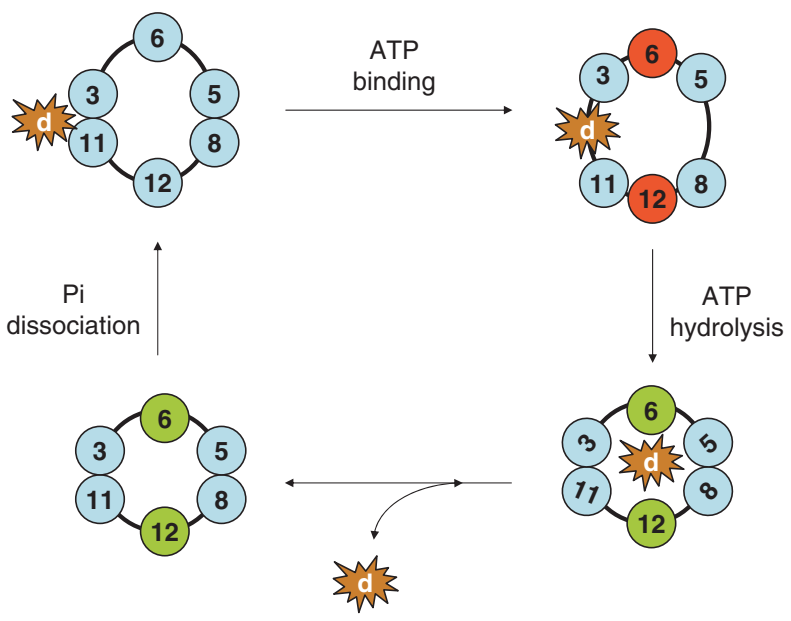

Fig. 2. The two-step model of drug translocation by ABCB1. (Top left) An arrangement of TM helices within the membrane-spanning domain of ABCB1. Numbered circles refer to specific helices and only a small selection are shown. The brown star-shaped object refers to a typical transported substrate of the protein. Altered colouration of the TM helices indicates that the segment has undergone a conformational change. A full description of the initial binding of drug to the high-affinity binding sites on $A B C B 1$ and the subsequent shift to a low-affinity site and a final dissociation to complete translocation across the membrane is given in the text. 
work using radiolabelled substrates suggests they are not involved. The working model of drug binding to ABCB1 (Fig. 2) will assume that they do not mediate drug binding, at least only the initial high-affinity step. They do, however, propagate TMD $\leftrightarrow$ NBD communication essential to coupling the translocation process. This model is based on (but not limited to) the hydrolysis of a single nucleotide per translocation event, as suggested by a number of excellent biochemical studies [42-44]. Moreover, the reader is directed to an earlier review for a more exhaustive mechanistic model on the complex sequence of events within the translocation mechanism of ABCB1 [45].

The other two helical pairs (TM3/11 and TM5/8) have been identified as binding drug using maleimidedrug conjugates, photo-cross-linking, mutagenesis and molecular modelling. Consequently, these helical pairs have been assigned as the initial drug-binding sites in the working model. Modelling and cross-linking data place these helices at the domain interface between the $\mathrm{N}$ - and C-terminal halves of the TMD. Drugs may bind to either TM3/11 or TM5/8 depending on their physicochemical properties, given the pharmacological data indicating multiple distinct sites in ABCB1.

The binding of ATP and the subsequent dimerization of the NBDs are believed to instigate the switch of the drug-binding sites from high to low affinity; i.e. the so-called 'power stroke' [46-50]. A recent article [51] further supports the data produced by Martin et al. that provided the original underlying evidence for a shift in the drug-binding site affinity in response to ATP binding at the NBDs [48]. The stimulus for this switch in ABCB1 is propagated through the TMDs via conformational changes in TM6/12, given their effects on the transport process and their direct contact with the NBDs. The consequence of this switch is that the drug now enters the central cavity. At this point, the TM helices lining the cavity (including TM6 and TM12) make contact with the bound drug, albeit with low affinity. This two-stage binding model therefore takes into account data implicating both the central cavity and domain interface models.

Further conformational changes caused by progression of the catalytic cycle result in the dissociation of drug from the central cavity and the final restoration of $\mathrm{ABCB} 1$ to its initial transport ready conformation. This model obviously requires further validation. However, it partially reconciles a substantial number of the available biochemical observations. In addition, it does not contravene the large number of studies that have described important characteristics of the drug-binding sites and their coupling to the NBDs.
How can we fully validate the two-step binding model? More biochemical data verifying the sites are of obvious importance, in particular, the cross-linking of drugs with subsequent digestion and MS analysis appears a powerful strategy. Structural data at high resolution ( $3 \AA$ or greater) would also be of invaluable assistance. Generating structural data in the presence of multiple drugs and protein in different conformations would provide the ideal information to fully elucidate the issue of drug binding to ABCB1. Such a goal remains elusive, but is clearly achievable given the recent published structure. Until then, however, the debate on the precise location of drug-binding sites in the protein will continue to rumble.

\section{Acknowledgements}

This investigation was generously supported by a Cancer Research UK Studentship to Emily Crowley (C362/A5502) for which Richard Callaghan was the principal investigator.

\section{References}

1 Ferry DR, Russell MA \& Cullen MH (1992) P-Glycoprotein possesses a 1,4-dihydropyridine selective drug acceptor site which is allosterically coupled to a vinca alkaloid selective binding site. Biochem Biophys Res Commun 188, 440-445.

2 Lugo MR \& Sharom FJ (2005) Interaction of LDS-751 and rhodamine 123 with P-glycoprotein: evidence for simultaneous binding of both drugs. Biochemistry $\mathbf{4 4}$, 14020-14029.

3 Martin C, Berridge G, Higgins CF, Mistry P, Charlton P \& Callaghan R (2000) Communication between multiple drug binding sites on P-glycoprotein. Mol Pharmacol 58, 624-632.

4 Orlowski S, Mir LM, Belehradek J \& Garrigos M (1996) Effects of steroids and verapamil on P-glycoprotein ATPase activity: progesterone, desoxycorticosterone and verapamil are mutually non-exclusive modulators. Biochem J 317, 515-522.

5 Bruggemann EP, Germann UA, Gottesman MM \& Pastan I (1989) Two different regions of P-glycoprotein [corrected] are photoaffinity-labeled by azidopine. $J$ Biol Chem 264, 15483-15488.

6 Greenberger LM (1993) Major photoaffinity labeling sites for iodoaryl azidoprazosin in P-glycoprotein are within or immediately C-terminal to transmembrane domains 6 and 12. J Biol Chem 268, 11417-11425.

7 Greenberger LM, Lisanti CJ, Silva JT \& Horwitz SB (1991) Domain mapping of the photoaffinity drug binding sites in P-glycoprotein encoded by mouse $m d r 1 \mathrm{~b}$. J Biol Chem 266, 20744-20751. 
8 Pleban K, Kopp S, Csaszar E, Peer M, Hrebicek T, Rizzi A, Ecker GF \& Chiba P (2005) P-Glycoprotein substrate binding domains are located at the transmembrane domain/transmembrane domain interfaces: a combined photoaffinity labelling-protein homology modeling approach. Mol Pharmacol 67, 365-374.

9 Devine SE, Ling V \& Melera PW (1992) Amino acid substitutions in the sixth transmembrane domain of P-glycoprotein alter multidrug resistance. Proc Natl Acad Sci USA 89, 4564-4568.

10 Kajiji S, Talbot F, Grizzuti K, Van Dyke-Phillips V, Agresti M, Safa AR \& Gros P (1993) Functional analysis of P-glycoprotein mutants identifies predicted transmembrane domain 11 as a putative drug binding site. Biochemistry 32, 4185-4194.

11 Loo TW \& Clarke DM (1993) Functional consequences of phenylalanine mutations in the predicted transmembrane domain of P-glycoprotein. J Biol Chem 268, 19965-19972.

12 Ma JF, Grant G \& Melera PW (1997) Mutations in the sixth transmembrane domain of P-glycoprotein that alter the pattern of cross-resistance also alter sensitivity to cyclosporin A reversal. Mol Pharmacol 51, 922-930.

13 Loo TW \& Clarke DM (2001) Determining the dimensions of the drug-binding domain of human P-glycoprotein using thiol cross-linking compounds as molecular rulers. J Biol Chem 276, 36877-36880.

14 Loo TW \& Clarke DM (2001) Defining the drug-binding site in the human multidrug resistance P-glycoycoprotein using a methanethiosulfonate analog of verapamil, MTS-verapamil. J Biol Chem 276, 1497214979.

15 Rothnie A, Storm J, Campbell J, Linton KJ, Kerr ID \& Callaghan R (2004) The topography of transmembrane segment six is altered during the catalytic cycle of P-glycoprotein. J Biol Chem 279, 34913-34921.

16 Storm J, O'Mara ML, Crowley EH, Peall J, Tieleman DP, Kerr ID \& Callaghan R (2007) Residue G346 in transmembrane segment six is involved in inter-domain communication in P-glycoprotein. Biochemistry 46, 9899-9910.

17 Dawson RJ \& Locher KP (2007) Structure of the multidrug ABC transporter Sav1866 from Staphylococcus aureus in complex with AMP-PNP. FEBS Lett 581, 935-938.

18 Ward A, Reyes CL, Yu J, Roth CB \& Chang G (2007) Flexibility in the ABC transporter MsbA: alternating access with a twist. Proc Natl Acad Sci USA 104, 19005-19010.

19 Khare D, Oldham ML, Orelle C, Davidson AL \& Chen J (2009) Alternating access in maltose transporter mediated by rigid-body rotations. Mol Cell 33, 528-536.

20 Lee J-Y, Urbatsch IL, Senior AE \& Wilkens S (2002) Projection structure of P-glycoprotein by electron microscopy. Evidence for a closed conformation of the nucleotide binding domains. $J$ Biol Chem 277, 4012540131.

21 Lee J-Y, Urbatsch IL, Senior AE \& Wilkens S (2008) Nucleotide-induced structural changes in P-glycoprotein observed by electron microscopy. J Biol Chem 283, 5769-5779.

22 Rosenberg MF, Callaghan R, Ford RC \& Higgins CF (1997) Structure of the multidrug resistance P-glycoprotein to $2.5 \mathrm{~nm}$ resolution determined by electron microscopy and image analysis. J Biol Chem 272, 10685-10694.

23 Rosenberg MF, Kamis AB, Callaghan R, Higgins CF \& Ford RC (2003) Three-dimensional structures of the mammalian multidrug resistance P-glycoycoprotein demonstrate major conformational changes in the transmembrane domains upon nucleotide binding. $J$ Biol Chem 278, 8294-8299.

24 Rosenberg MF, Velarde G, Ford RC, Martin C, Berridge G, Kerr ID, Callaghan R, Schmidlin A, Wooding C, Linton KJ et al. (2001) Repacking of the transmembrane domains of P-glycoprotein during the transport ATPase cycle. EMBO $J$ 20, 5615-5625.

25 Loo TW \& Clarke DM (2005) Recent progress in understanding the mechanism of P-glycoprotein-mediated drug efflux. J Membr Biol 206, 173-185.

26 Loo TW, Bartlett MC \& Clarke DM (2004) Disulfide cross-linking analysis shows that transmembrane segments 5 and 8 of human P-glycoprotein are close together on the cytoplasmic side of the membrane. J Biol Chem 279, 7692-7697.

27 Loo TW \& Clarke DM (2000) Identification of residues within the drug binding domain of the human multidrug resistance P-glycoprotein by cysteine-scanning mutagenesis and reaction with dibromobimane. $J$ Biol Chem 275, 39272-39278.

28 Loo TW, Bartlett MC \& Clarke DM (2003) Methanethiosulfonate derivatives of rhodamine and verapamil activate human P-glycoprotein at different sites. $J$ Biol Chem 278, 50136-50141.

29 Crowley E, O'Mara ML, Reynolds C, Tieleman DP, Storm J, Kerr ID \& Callaghan R (2009) Transmembrane helix 12 modulates progression of the ATP catalytic cycle in ABCB1. Biochemistry 48, 6249-6258.

30 Storm J, Modok S, O'Mara ML, Tieleman DP, Kerr ID \& Callaghan R (2008) Cytosolic region of TM6 in P-glycoprotein: topographical analysis and functional perturbation by site directed labeling. Biochemistry 47, 3615-3624.

31 Locher KP, Lee AT \& Rees DC (2002) The E. coli BtuCD structure: a framework for ABC transporter architecture and mechanism. Science 296, 1091-1098.

32 Oldham ML, Khare D, Quiocho FA, Davidson AL \& Chen J (2007) Crystal structure of a catalytic intermediate of the maltose transporter. Nature 450, 515521. 
33 Becker JP, Depret G, Van Bambeke F, Tulkens PM \& Prevost M (2009) Molecular models of human P-glycoprotein in two different catalytic states. BMC Struct Biol 9, 3, doi:10.1186/1472-6807-9-3.

34 O'Mara ML \& Tieleman DP (2007) P-Glycoprotein models of the apo and ATP-bound states based on homology with Sav1866 and MalK. FEBS Lett 581, 4217-4222.

35 Ravna AW, Sylte I \& Sager G (2007) Molecular model of the outward facing state of the human P-glycoprotein (ABCB1), and comparison to a model of the human MRP5 (ABCC5). Theor Biol Med Model 4, 33, doi:10.1186/1742-4682-4-33.

36 Vandevuer S, Van Bambeke F, Tulkens PM \& Prevost M (2006) Predicting the three-dimensional structure of human P-glycoprotein in absence of ATP by computational techniques embodying crosslinking data: insight into the mechanism of ligand migration and binding sites. Proteins Struct Funct Bioinform 63, 466-478.

37 Al-Shawi MK \& Senior AE (1993) Characterization of the adenosine triphosphatase activity of Chinese hamster P-glycoprotein. J Biol Chem 268, 4197-4206.

38 Martin C, Berridge G, Mistry P, Higgins C, Charlton P \& Callaghan R (1999) The molecular interaction of the high affinity reversal agent XR9576 with P-glycoprotein. Br J Pharmacol 128, 403-411.

39 Sharom FJ (1995) Characterization and functional reconstitution of the multidrug transporter. $J$ Bioenerg Biomembr 27, 15-22.

40 Safa AR, Stern RK, Choi K, Agresti M, Tamai I, Mehta ND \& Roninson IB (1990) Molecular basis of preferential resistance to colchicine in multidrug resistant human cells conferred by Gly185-Val185 substitution in P-glycoprotein. Proc Natl Acad Sci USA 87, 7225-7229.

41 Glossmann H, Ferry DR, Striessnig J, Goll A \& Moosburger K (1987) Resolving the structure of the $\mathrm{Ca}^{2+}$ channel by photoaffinity labeling. Trends Pharmacol Sci 8, 95-100.

42 Carrier I, Julien M \& Gros P (2003) Analysis of catalytic carboxylate mutants E552Q and E1197Q suggests asymmetric ATP hydrolysis by the two nucleotide-binding domains of P-glycoprotein. Biochemistry 42, 1287512885.

43 Urbatsch IL, Sankaran B, Weber J \& Senior AE (1995) P-Glycoprotein is stably inhibited by vanadate-induced trapping of nucleotide at a single catalytic site. $J$ Biol Chem 270, 19383-19390.

44 Urbatsch IL, Tyndall GA, Tombline G \& Senior AE (2003) P-Glycoprotein catalytic mechanism: studies of the ADP-vanadate inhibited state. J Biol Chem 278, 23171-23179.

45 Callaghan R, Ford RC \& Kerr ID (2006) The translocation mechanism of P-glycoprotein. FEBS Lett 580, 1056-1063.
46 Higgins CF \& Linton KJ (2004) The ATP switch model for ABC transporters. Nat Struct Mol Biol 11, 918-926.

47 Maki N, Moitra K, Ghosh P \& Dey S (2006) Allosteric modulation bypasses the requirement for ATP hydrolysis in regenerating low affinity transition state conformation of human P-glycoprotein. J Biol Chem 281, 10769-10777.

48 Martin C, Higgins CF \& Callaghan R (2001) The vinblastine binding site adopts high- and low-affinity conformations during a transport cycle of P-glycoprotein. Biochemistry 40, 15733-15742.

49 Sauna ZE, Nandigama K \& Ambudkar SV (2006) Exploiting reaction intermediates of the ATPase reaction to elucidate the mechanism of transport by P-glycoprotein (ABCB1). J Biol Chem 281, 26501-26511.

50 Abele R \& Tampe R (2004) The ABCs of immunology: structure and function of TAP, the transporter associated with antigen processing. Physiology (Bethesda) 19, 216-224.

51 Aanismaa P, Gatlik-Landwojtowicz E \& Seelig A (2008) P-Glycoprotein senses its substrates and the lateral membrane packing density: consequences for the catalytic cycle. Biochemistry 47, 10197-10207.

52 Greenberger LM, Yang C-PH, Gindin E \& Horwitz SB (1990) Photoaffinity probes for the $\mathrm{a}_{1}$-adrenergic receptor and the calcium channel bind to a common domain in P-glycoprotein. J Biol Chem 265, 4394-4401.

53 Pawagi AB, Wang J, Silverman M, Reithmeier RA \& Deber CM (1994) Transmembrane aromatic amino acid distribution in P-glycoprotein. A functional role in broad substrate specificity. J Mol Biol 235, 554-564.

54 Zhang X, Collins KI \& Greenberger LM (1995) Functional evidence that transmembrane 12 and the loop between transmembrane 11 and 12 form part of the drug-binding domain in P-glycoycoprotein encoded by MDR1. J Biol Chem 270, 5441-5448.

55 Dey S, Ramachandra M, Pastan I, Gottesman MM \& Ambudkar SV (1997) Evidence for two nonidentical drug-interaction sites in the human P-glycoycoprotein. Proc Natl Acad Sci USA 94, 10594-10599.

56 Seelig A (1998) A general pattern for substrate recognition by P-glycoprotein. Eur J Biochem 251, 252 261.

57 Hafkemeyer P, Dey S, Ambudkar SV, Hrycyna CA, Pastan I \& Gottesman MM (1998) Contribution to substrate specificity and transport of nonconserved residues in transmembrane domain 12 of human P-glycoprotein. Biochemistry 37, 16400-16409.

58 Isenberg B, Thole H, Tummler B \& Demmer A (2001) Identification and localization of three photobinding sites of iodoarylazidoprazosin in hamster P-glycoprotein. Eur J Biochem 268, 2629-2634.

59 Song J \& Melera PW (2001) Transmembrane domain (TM) 9 represents a novel site in P-glycoprotein that affects drug resistance and cooperates with TM6 to 
mediate $\left[{ }^{125} \mathrm{I}\right]$ iodoarylazidoprazosin labeling. Mol Pharmacol 60, 254-261.

60 Lugo MR \& Sharom FJ (2005) Interaction of LDS-751 with P-glycoprotein and mapping of the location of the R drug binding site. Biochemistry 44, 643-655.

61 Loo TW \& Clarke DM (2005) Do drug substrates enter the common drug-binding pocket of P-glycoprotein through 'gates'? Biochem Biophys Res Commun 329, 419-422.

62 Loo TW, Bartlett MC \& Clarke DM (2006) Transmembrane segment 7 of human P-glycoprotein forms part of the drug-binding pocket. Biochem $J$ 399, 351359.

63 Loo TW, Bartlett MC \& Clarke DM (2006) Transmembrane segment 1 of human P-glycoprotein contributes to the drug-binding pocket. Biochem $J$ 396, 537-545.

64 Omote H \& Al-Shawi MK (2006) Interaction of transported drugs with the lipid bilayer and P-glycoprotein through a solvation exchange mechanism. Biophys $J \mathbf{9 0}$, 4046-4059. 